\title{
An Algorithm for the Location of Branching Points
}

\section{on Reaction Paths}

\author{
J. Baker \\ Department of Theoretical Chemistry, University Chemical Laboratory, Lensfield Road, \\ Cambridge CB2 1EW, England
}

P. M. W. Gill

Research School of Chemistry, Australian National University, Canberra ACT 2601, Australia

Received 11 June 1987; accepted 23 November 1987

\begin{abstract}
A branching point is a point on a reaction path leading from reactants to products (via a transition state) at which it is energetically favorable for the system to break symmetry. Such a point can be defined in terms of normal modes along the reaction path and corresponds to zero curvature (a zero Hessian eigenvalue) along a symmetry-breaking mode. An effective method for the location of such points is presented and realized in an efficient, practical algorithm designed for use in the $a b$ initio program package Gaussian 82 .
\end{abstract}

\section{INTRODUCTION}

A useful picture of how a chemical reaction proceeds that is familiar to most chemists is one where reactants and products lie in "wells" on potential energy surfaces, with the reactant transforming into the product by generally taking the lowest energy pathway available to it which leads from the reactant "well" to the product "well," passing through a transition state - the highest energy point on this pathway - as it does so. Implicit in this picture is the concept of the "reaction path," that is, how the various atoms in the reactant molecules alter their positions with respect to one another-changes in bond lengths and bond angles - as the reaction proceeds. Although obviously an oversimplification - given enough energy reactant molecules can wander all over the potential surface and even leave it altogether - this concept is very appealing and is certainly reasonable as a "zeroth order" approximation.

Perhaps the best way of defining such a reaction path is by means of the intrinsic reaction coordinate (IRC), first proposed in this context by Fukui. ${ }^{1}$ Basically this path is the union of the two steepest descent paths leading from the transition state down to the reactant and product wells, respectively. The definition is unambiguous since exactly one steepest descent path originates in either downhill direction at the transition state, its initial direction being the same as that of the eigenvector of the Hessian (the second derivative matrix) corresponding to the negative eigenvalue. The first practical algorithm for following the IRC down from a transition state to a minimum was given by Ishida et al. ${ }^{2}$

\section{FOLLOWING A TRANSITION STATE DOWNHILL}

If the eigenvector corresponding to the negative eigenvalue in the force constant matrix for a transition state is followed smoothly downhill, then the curvature for this particular mode (normal coordinate) along the lowest energy path will change from being negative (in the region of the transition state) to positive (in the region of the minimum). During this motion all other eigenvalues of the Hessian must remain positive; if one of the eigenvalues became negative it would mean that it is possible to move downhill (i.e., to lower the energy) along the corresponding Hessian mode and the system would no longer be on the lowest energy path. Thus the lowest energy path downhill from a transition state must lead to a minimum. 
It may of course be possible to find a constrained path connecting two transition states. For such a path, the curvature of the energy surface would change from negative to positive along the mode being followed downhill, but from positive to negative along some other mode, motion along the second mode being prevented by, for instance, symmetry constraints. A schematic of the energy surface in such a situation is shown in Figure 1. Here T1 is a minimum in all directions (normal coordinates) except one (it is a maximum in the plane of the paper say). Along the path (shown by the solid curve) from T1 to T2 the curvature in this direction changes from negative to positive and $\mathrm{T} 2$ is a minimum along this normal coordinate. However, perpendicular to the plane of the paper T2 is a maximum. Along the "valley path" joining T1 and $\mathrm{T} 2$ the valley gradually widens until it is locally virtually flat; thereafter it begins to curve in the opposite direction and turns into a ridge. The point $B$ on this pathway represents a "branching" or "bifurcation" point, where the curvature along some direction other than the one being followed changes from positive to negative. At B, this curvature would be zero. Thereafter the lowest energy pathway would follow the dashed line to M1 or M2. The ridge from B to T2 would only be followed under a suitably rigorous constraint, allowing no motion along this second direction (i.e., off the ridge). It is with the location of such branching points that this article is concerned.

Note that, strictly speaking, the IRC path down from $\mathrm{T} 1$ goes through $\mathrm{B}$ to $\mathrm{T} 2$, in other words, it will not break symmetry. However, after the branching point the IRC is no longer the lowest energy path and hence is no longer useful as a definition of the reaction path.

There has been a fair amount of discussion in the literature over the last five years or so on potential energy surfaces and reaction paths. A useful introduction to the concepts involved is given in the 1980 review by Muller, ${ }^{3}$ although from a practical point of view this is a little out of date now. Basilevsky and Shamov ${ }^{4}$ have presented an algorithm for climbing uphill from a minimum to a transition state (the "mountaineer's algorithm") although the "optimum ascent path" mapped

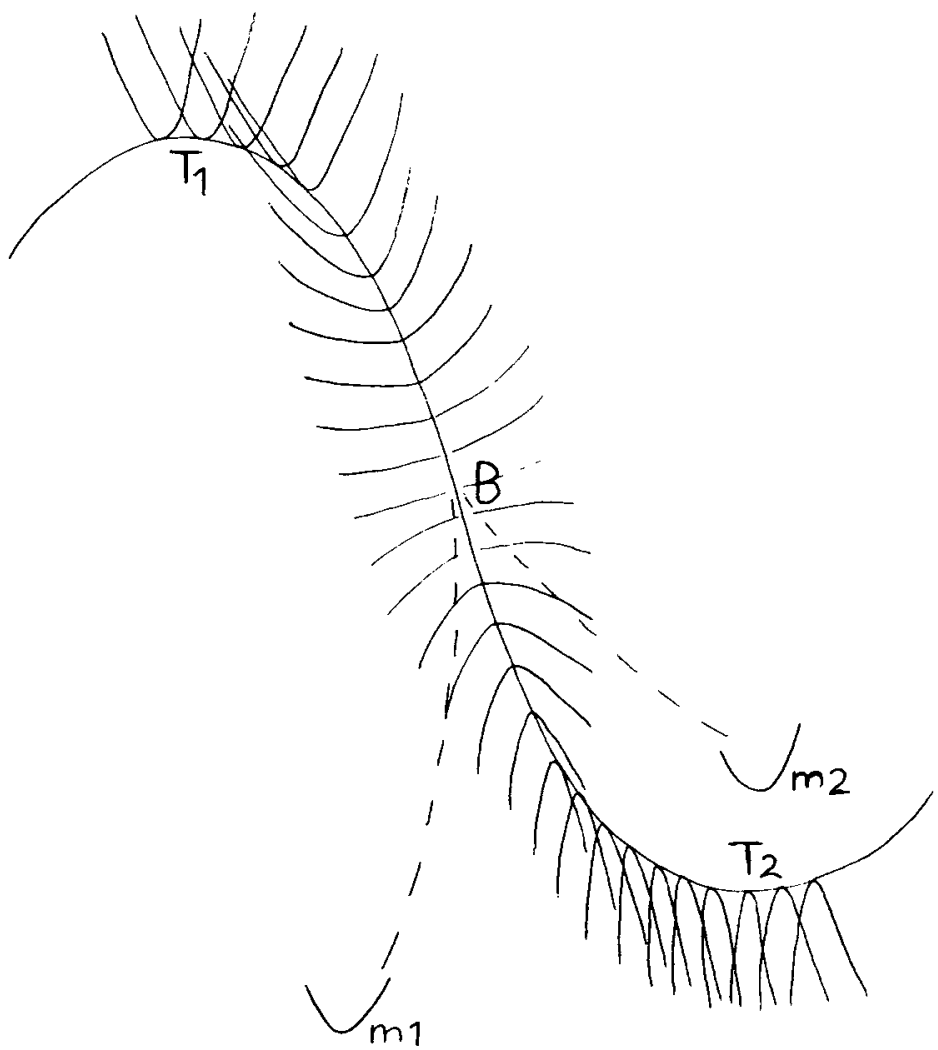

Figure 1. A constrained reaction path connecting two transition states. The solid line joining $\mathrm{T} 1$ and $\mathrm{T} 2$ is a symmetry-conserving pathway. B represents the branching point on this reaction path; M1 and M2 are minima (see the text for more details). 
out by their approach does not, in general, coincide with the IRC path. Two recent papers of interest are those of Hoffman et al. ${ }^{5}$ and Valtazanos and Ruedenberg ${ }^{6}$; these are directly concerned with branching points (which they term "valley-ridge inflection points").

\section{COORDINATE SYSTEMS AND THE HESSIAN}

One area of possible confusion in the literature regarding minimum energy pathways is with the coordinate system chosen to map out the path. Most authors use mass-weighted cartesian coordinates and both Fukui's original definition of the IRC and Ishida et al.'s algorithm ${ }^{2}$ use this coordinate system. It is of course perfectly possible to define a reaction path in any coordinate system; an obvious candidate would be a set of (in general) $3 \mathrm{~N}-6$ internal coordinates (where $\mathrm{N}$ is the number of atoms). However, it should be noted that a reaction path based on a steepest descent in a given internal coordinate system will be different to a similar path calculated in another internal coordinate system and both paths will be different to the steepest descent path calculated in cartesian coordinates (massweighted or otherwise). Thus - for consistency, if nothing else - it is advisable to pick one particular coordinate system and define the reaction path in this system and massweighted cartesians are the obvious choice.

Reaction paths in internal coordinates can be related to reaction paths in mass-weighted cartesians by suitable transformations involving the Wilson $\mathbf{G}$ matrix $^{7}$; Sana et al. ${ }^{8}$ have presented an algorithm which does just this. They claim that their algorithm provides a general way of following Fukui's IRC in any set of $3 \mathrm{~N}-6$ internal coordinates. Transforming the reaction path in this way is not useful for our purposes, however, since for our algorithm we need to know curvatures and normal modes at points along the reaction path and this would require a transformation of the Hessian. The only really satisfactory way to give a coordinate-free definition of the Hessian at any point on the potential energy surface is to use differential geometry, as has been discussed by Tachibana and Fukui. ${ }^{9}$ In any case, since these transformations relate quantities in internal coordinates to the same quantities in mass-weighted cartesians it seems sensible to dispense with the transformations and use mass-weighted cartesians from the start.

The use of mass-weighted cartesians does pose a minor problem to do with the Hessian. As mentioned above, at any given point on the reaction path we require a direction (normal mode) downhill which we are following and $3 \mathrm{~N}-7$ normal modes orthogonal to this defining curvatures along directions perpendicular to the reaction path (the branching point corresponds to zero curvature - a zero Hessian eigenvalue - along one of these modes). Thus the six modes in cartesian coordinates corresponding to infinitesimal translations and rotations have to be "removed." In addition, the usual normal mode analysis used to characterize an optimized structure as a minimum, a transition state, or whatever, is only valid at a stationary point, namely at a point at which the gradient is zero, which it certainly is not at an arbitrary point along the reaction path. Neither of these points is a problem in internal coordinates.

Both of these problems can be overcome by using the projected force constant matrix of Miller et al. ${ }^{10}$ In this approach normal modes for vibration along the reaction path are defined by diagonalizing the projected force constant matrix

$$
\mathbb{H}^{P}=(1-\mathbb{P}) \mathbb{H}(1-\mathbb{P})
$$

where $H$ is the force constant matrix in cartesian coordinates and $P$ is a projection matrix defined by

$$
\mathbb{P}=\sum_{i=1}^{7} \mathbf{V}_{i} \mathbf{V}_{i}^{\vdash}
$$

where the $V_{i}$ are the six eigenvectors of $H$ corresponding to the translations and rotations plus the eigenvector corresponding to the downhill direction, that is the IRC mode.

Strictly speaking we do not form the projected force constant matrix in precisely the same way as Miller et al.; what is actually done is to project out the gradient, that is, to use as the projection matrix

$$
\mathbb{P}=\mathbf{g} \cdot \mathbf{g}^{+}
$$

where $\mathbf{g}$ is the (normalized) gradient vector, and to perform a normal mode analysis on the resulting force constant matrix (the normal mode analysis has the projection of the trans- 
lations and rotations built into it). In essence, this is the same as the Miller projection since, if we are accurately following the IRC, that is, we are at the bottom of the valley path, the IRC mode points in exactly the same direction as the gradient - the gradient is an eigenvector of the projected Hessian. In practice, of course, the two directions will only be approximately the same.

The basic idea behind projecting out the gradient is that, with the linear term in the Taylor series expansion of the energy removed, the system can be treated as if it were lying at the bottom of a harmonic well and a standard normal mode analysis can be performed. Projecting out the gradient in this way results in the IRC mode having a zero eigenvalue. This cannot be confused with the zero eigenvalue at the branching point, since one of these modes breaks symmetry while the other does not. Note that it is not really necessary to project out the gradient at all; modes that break symmetry are unaffected by the projection and the gradient vector itself can be taken as the IRC mode. However, without projecting, curvatures along all the other modes are meaningless.

\section{INTRODUCTION TO THE ALGORITHM}

In order to expand on some of the concepts involved in the location of branching points, which are really fairly simple, it might be instructive to take an actual example and for this purpose we consider the isomerization of the methoxy radical

$$
\mathrm{CH}_{3} \mathrm{O} \longleftrightarrow \mathrm{CH}_{2} \mathrm{OH}
$$

This isomerization was the subject of a reaction path Hamiltonian study by Colwell ${ }^{11}$ and is known to possess a branching point. The part of the potential surface of interest for our purposes is shown schematically in Figure 2 (at the STO-3G level). The relevant geometrical structures are given in Figure 3.

Structure 1 is the transition state (TS) for the isomerization. This solid curve linking 2 and 3 via 1 represents a symmetryconserving pathway as all three structures 1 , 2 , and 3 have $C_{s}$ symmetry. However, 2 is not a true minimum; it has one imaginary frequency and is, in fact, the TS linking two equivalent $C_{1}$ structures 2 ' which is the local

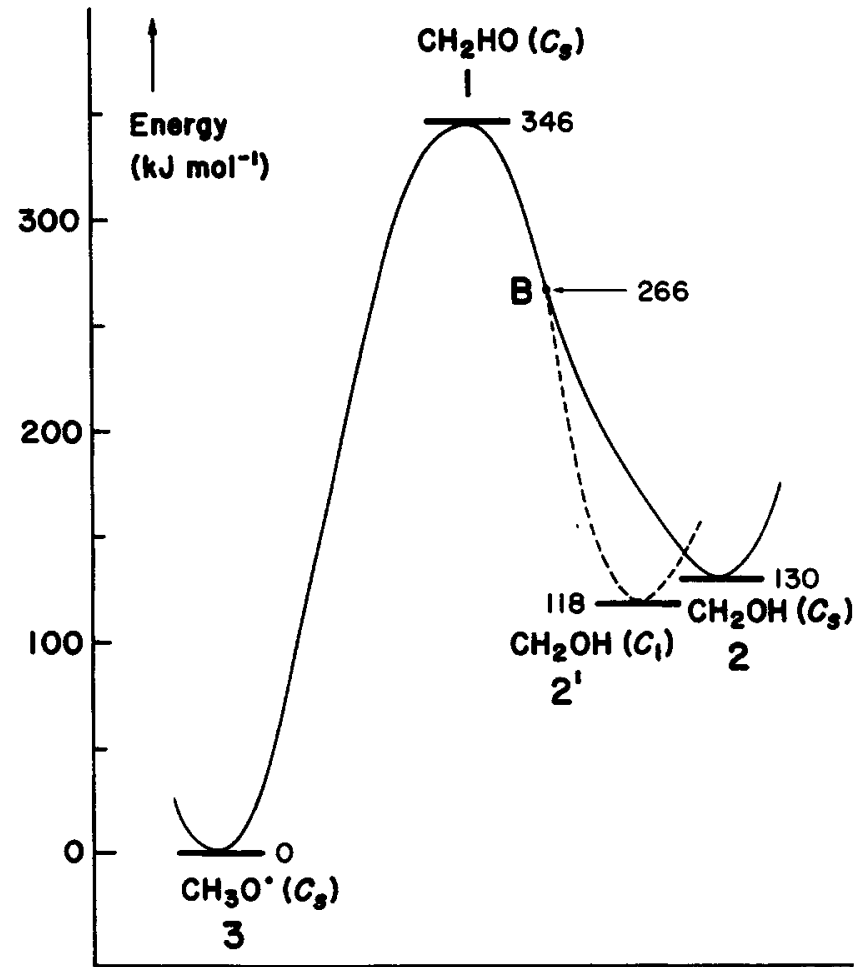

Figure 2. The $\mathrm{CH}_{3} \mathrm{O}$ potential energy surface at the UHF/STO-3G level. Relative energies are in $\mathrm{kJ} \mathrm{mol}^{-1}$.

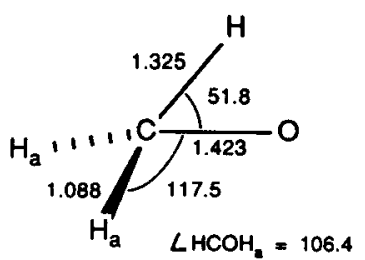

$1 C_{s}$ Transition State

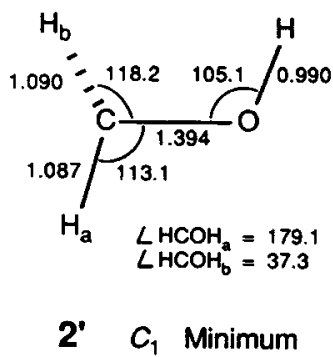

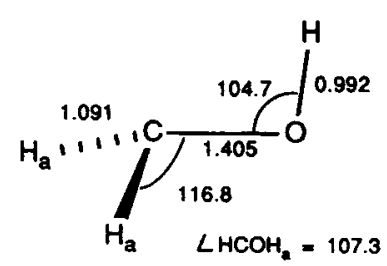

$2 C_{s}$ "False" Minimum

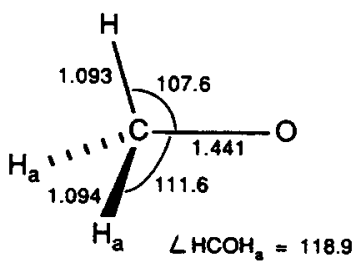

$3 \quad c_{s}$ Minimum

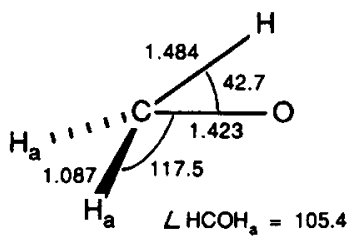

B $C_{s}$ Branching Point

Figure 3. Stationary and branching points on the $\mathrm{CH}_{3} \mathrm{O}$ potential energy surface. Bond lengths in $\mathrm{A}$; bond angles in degrees. 
minimum in this region of the energy surface. 2 ' lies $12 \mathrm{~kJ} \mathrm{~mol}^{-1}$ below 2 . Somewhere on the $\mathrm{C}_{s}$ pathway between 1 and 2 the system will break $\mathrm{C}_{s}$ symmetry in order to reach $2^{\prime}$; this point is designated as point $\mathrm{B}$ on the solid $\left(\mathrm{C}_{s}\right)$ curve linking 1 and 2 and represents the branching point on this reaction path.

One way of obtaining an estimate of the location of the branching point is to follow the reaction path down from 1 toward 2 and periodically determine the curvatures and normal modes (as outlined in the previous section) at various points along the path. Note that, whereas the reaction path and the branching point, have $\mathrm{C}_{s}$ symmetry, the force constant matrix (from which the curvatures are obtained) must be evaluated in a coordinate system that permits a $\mathrm{C}_{s}-\mathrm{C}_{1}$ symmetry breaking; if the Hessian is evaluated in a strictly $\mathrm{C}_{s}$ coordinate system the branching point will never be found as no Hessian mode will ever break $\mathrm{C}_{s}$ symmetry. Thus the Hessian should be allowed all $3 \mathrm{~N}-6$ degrees of freedom. A plot of the lowest symmetrybreaking Hessian eigenvalue (curvature) versus distance down the reaction path from 1 to 2 (in mass-weighted cartesians) is shown in Figure 4. As can be seen the curvature along the symmetry-breaking mode changes smoothly from a value of +0.03113 at the TS to -0.00501 at a distance of ca. 074 a.u. down

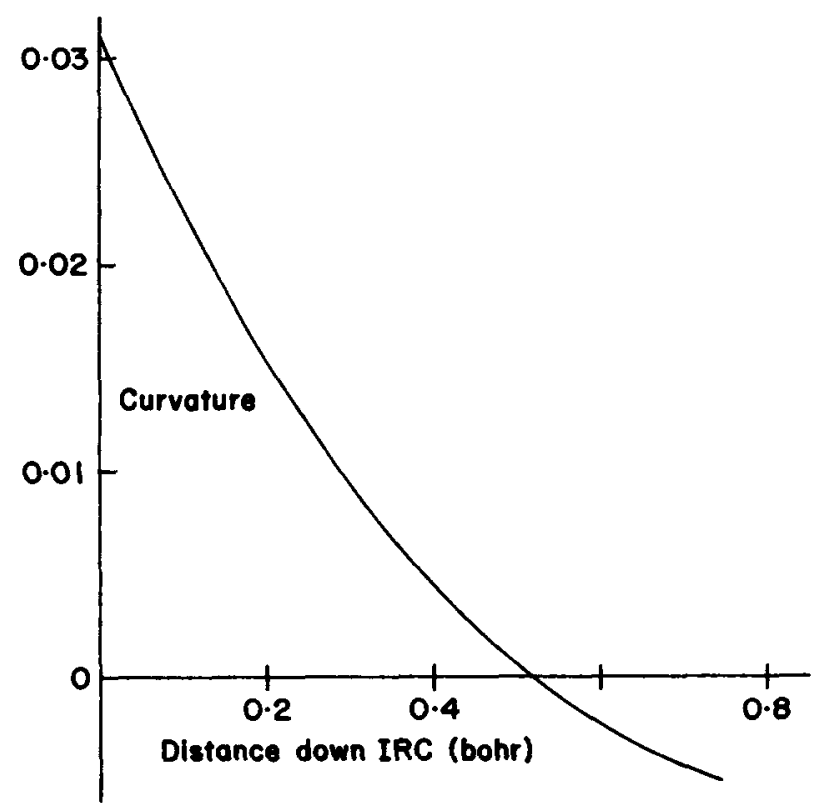

Figure 4. Curvature along symmetry-breaking mode versus distance down the IRC path from the transition state (in mass-weighted cartesian coordinates; all values in atomic units) for the isomerization of the methoxy radical. the IRC; zero curvature - the branching point-occurs at a distance of ca. 0.58 a.u. down the IRC, where the curve crosses the horizontal axis.

By essentially assuming the behavior outlined above, that is, a smooth change in the curvature along the symmetry-breaking mode from positive through zero to negative along the reaction path, the algorithm attempts to predict the distance from the current point to the branching point and "walks" down the reaction path the predicted distance. The Hessian is then reevaluated at the new point, a new prediction made and so on until a preset convergence criterion on the zero Hessian eigenvalue is attained or until the search is terminated for some other reason.

The algorithm can be thought of as being in two parts: Firstly, after calculating the Hessian at a particular point along the reaction path, predicting how far away the branching point is from the current point and secondly, "walking" the predicted distance on the potential energy surface while ensuring that the system remains on the reaction path.

For the second part we use essentially the IRC algorithm of Ishida et al. ${ }^{2}$ Details of this algorithm are well known and will not be repeated here. For the line search needed to get back onto the IRC path following each steepest descent step we make use of the projected gradient, as suggested by Schmidt et al. ${ }^{12}$

To estimate the distance to the branching point we proceed as follows: Typically the branch point search will commence from the transition state (this is not a prerequisite, the search can be started from any point on the reaction path above the branching point, but unless a preliminary path has been "mapped out" beforehand the only point definitely known to lie on the reaction path will be the TS). In most circumstances the Hessian will already be available, having been calculated in order to characterize the stationary point as a TS, but if not, it should be evaluated. Two of the Hessian modes are then chosen; one, the IRC mode, the mode to be followed downhill (this will simply be the mode with negative curvature), and the other the perpendicular or symmetry-breaking mode (this will generally be the lowest mode that breaks symmetry). The curvature along the perpendicular mode is noted. A finite- 
difference step is then taken downhill along the direction of the IRC mode. From the new point a second finite-difference step is taken along the direction of the perpendicular mode. This step will break symmetry. A parabola is fitted through these two points, assuming that the point along the IRC mode is the minimum/maximum of the parabola. This then gives an estimate of the curvature in the direction of the perpendicular mode at a point slightly downhill from the starting point. If the energy after taking IRC and perpendicular finite-difference steps of size FDSTEP is $\mathrm{E} 0$ and $\mathrm{E} 1$, respectively, the curvature is given by 2(E1-E0)/FDSTEP. This procedure is then repeated, in other words, returning to the point following the initial IRC finitedifference step, another finite-difference step is taken followed by another perpendicular finite-difference step and a new curvature obtained. With three estimates of the curvature at (roughly) three points along the reaction path a quadratic is fitted through these three points and extrapolated to zero curvature, the distance to which represents an estimate of the distance from the current point to the branching point. This entire process is termed the quadratic extrapolation step. If the quadratic has two positive roots, the lowest root is taken; if it has two negative or imaginary roots the quadratic extrapolation is rejected in favor of a simple linear fit; if this also fails (which will be the case if the curvature along the perpendicular mode increases instead of decreasing) then an arbitrary maximum distance DTMAX (default 0.6 a.u.) is taken. If the predicted distance to the branching point is greater than this maximum it is reduced.

Having obtained an estimate of the distance to the branching point the algorithm proceeds to walk this distance downhill along the reaction path (the IRC). During the walk the following restrictions are imposed on the IRC search:

1. Wherever possible the steplength taken along the IRC should not be less than DMIN (default 0.005 a.u.).

2. At least three steps should be taken subject to (1).

3. No steplength should exceed DMAX (default 0.15 a.u.).

The reasons for the above restrictions, particularly (2), will become apparent later. As examples of how they would apply then for a predicted distance of 0.6 a.u., four steps would be taken each of size 0.15 (DMAX); for 0.3 a.u., three steps would be taken each of size 0.1 and for 0.01 a.u. two steps would be taken of size 0.005 (DMIN).

On arrival at the estimated branching point, the Hessian is reevaluated and the new IRC and perpendicular modes are determined. This can be done by criterion of maximum overlap with the previously selected modes or by selecting the mode having the greatest overlap with the gradient vector (the IRC mode) and the lowest mode that breaks symmetry (the perpendicular mode). Depending on how far down the reaction path we have travelled the curvature along either mode may be positive or negative. If the curvature along the perpendicular mode remains positive then exactly the same procedure as before is repeated: a series of finite-difference steps followed by quadratic extrapolation on the estimated curvatures. Since the gradient is no longer zero a steepest descent finitedifference step can be taken instead of a step in the direction of the IRC mode (as mentioned in the previous section, the two directions should in theory be the same); however in the majority of cases tested to date the IRC step results in a better prediction for the location of the branching point. If the curvature is negative then we have overshot the branch point and we have to go back. Since an ascent path following the gradient is numerically unstable (i.e., we can only go down the IRC and not up), what is actually done is to go back to a suitable point on the IRC calculated during the original descent and then walk down the required distance. During the IRC search all points calculated on the IRC path and their gradients are stored so it is a simple matter to go back to a suitable point. It is for this reason that at least three IRC steps are taken between each estimated branching point; this ensures that there are a choice of points to go back to if necessary. Once a point with negative curvature has been reached this confines the search to a particular region of the potential energy surface; the branch point must occur before that point.

The estimated distance back along the IRC path from a point with negative curvature can be found in exactly the same way as before using quadratic extrapolation; however, 
an attempt is first made at a simple linear interpolation between the last point with positive curvature and the current point. If the predicted distance is greater than the distance to the previous point lying on the IRC, then the interpolation is rejected in favor of the usual quadratic extrapolation; if it is less then we return to the last point on the IRC and walk back down to the branching point as explained in the previous paragraph.

The above presents in some detail the essential features of the branch point algorithm. A series of IRC steps and Hessian evaluations is carried out until a point is located on the reaction path with zero curvature along the perpendicular mode to within a given tolerance (default $10^{-5}$ a.u.) or until the estimated distance to the branch point is insignificant (default 0.001 a.u.). For the test reaction studied here, starting from the transition state, convergence on the Hessian eigenvalue was achieved after five Hessian evaluations, including the Hessian at the TS which was already available. The final geometry of the branching point is shown in Figure 3 and its relative energy given on Figure 2. The accuracy in determining the branching point appears to be excellent; starting the search from various points down the IRC instead of at the TS makes no real difference to the final parameter values, the convergence criterion on the Hessian eigenvalue is such that the same kind of accuracy as in a normal geometry optimization is attained. The accuracy on the energy is not so great, which is not surprising considering that the branching point does not lie at the bottom of a potential well, and depends on the "steepness" of the energy surface near the branching point; for this system it is within $5 \times 10^{-5}$ Hartree which is really very good.

Before presenting the algorithm in full we consider another example of a branching point on the reaction path for the rearrangement

$$
\mathrm{H}_{2} \mathrm{CO} \longleftrightarrow \mathrm{HCOH}
$$

at the STO-3G level. A schematic of the relevant portion of the potential energy surface is shown in Figure 5. The geometrical structures are given in Figure 6.

The solid line linking structures 2 and 3 and passing through 1 is a symmetry preserving pathway which preserves a $\mathrm{C}_{s}$ symmetry plane. However 1 is not the true

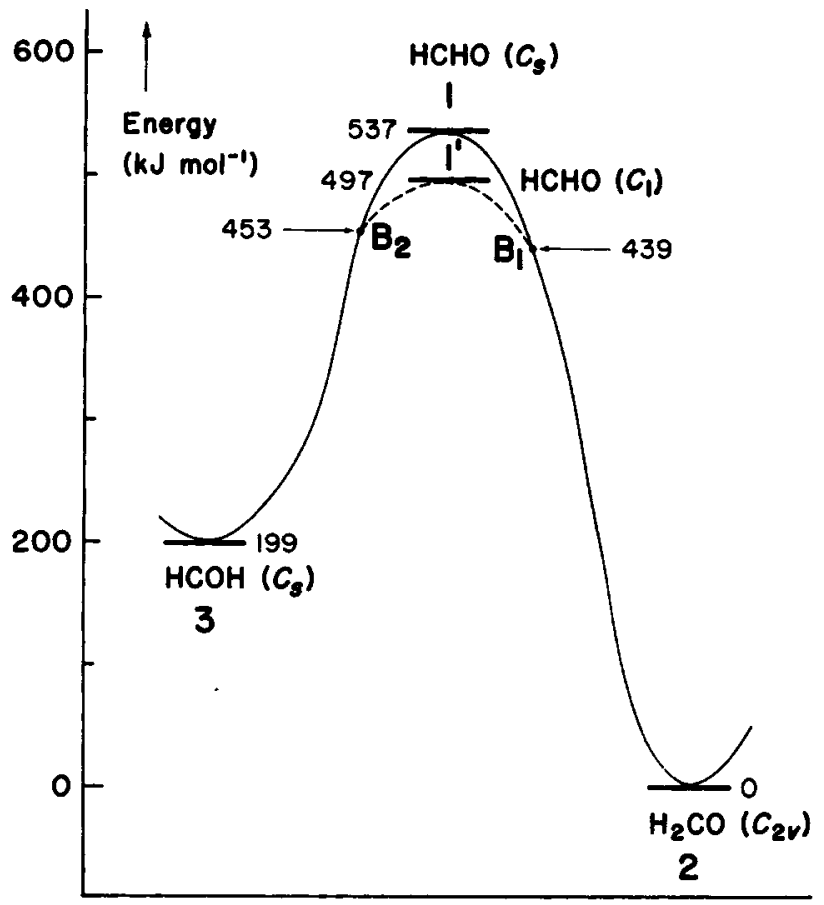

Figure 5. The $\mathrm{H}_{2} \mathrm{CO}$ potential energy surface at the $\mathrm{RHF} / \mathrm{STO}-3 \mathrm{G}$ level. Relative energies are in $\mathrm{kJ} \mathrm{mol}^{-1}$.

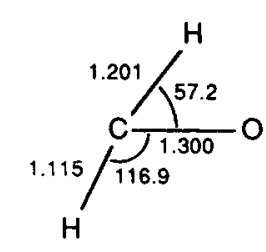

1

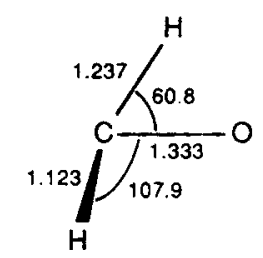

$c_{s}$ "False" Transition
State

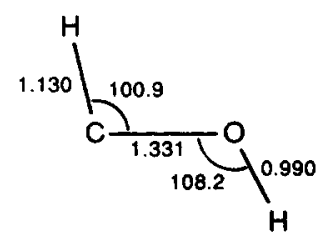

$2 \quad c_{s}$ Minimum

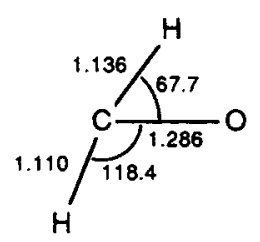

B $C_{s}$ Branching Point

Figure 6. Stationary and branching points on the $\mathrm{H}_{2} \mathrm{CO}$ potential energy surface. Bond lengths in $\mathrm{A}$; bond angles in degrees. 
transition state for this rearrangement as it has two imaginary frequencies; the real TS is $1^{\prime}$ which has $\mathrm{C}_{1}$ symmetry (structure 1 can be thought of as linking two equivalent structures 1' with a hydrogen atom bent in front of and behind the plane of the paper, respectively). Thus somewhere on the path from 2 to $1 \mathrm{C}_{s}$, symmetry will be lost and a branching point will occur; similarly there will be another branching point on the $\mathrm{C}_{s}$ path from 3 to 1.

These branching points cannot be easily located by starting from $1^{\prime}$, the true TS, as this has no symmetry. Instead we start from 1 and follow the symmetry-preserving Hessian mode corresponding to one of the negative eigenvalues, the other mode with negative curvature necessarily being the symmetry breaking mode. Thus in this case, unlike in our first example, the curvature along the perpendicular mode starts off being negative and changes to positive along the IRC path, that is, we are going down the top of a ridge which transforms into a valley rather than moving down a valley which changes into a ridge.

The two branching points on the surface can be located in essentially the same way as before; their geometries are included in Figure 6 and relative energies on Figure 5. B1 was located in five Hessian evaluations and B2 in three.

\section{THE ALGORITHM}

As with the algorithms for analytical ${ }^{13}$ and numerical $^{14}$ geometry optimization previously published in this journal, the branch point algorithm has been made completely compatible with and "built in" to the $a b$ initio program package Gaussian $82 .^{15}$ Below we give a description of the main steps involved in the algorithm within the framework of Gaussian 82.

1. Select the coordinate system used to describe the reaction path. This will normally be mass-weighted cartesians (default) but cartesian coordinates (giving a mass-independent path) or a set of $3 N-6$ internal coordinates can be chosen.

2. Start the search from a point known to lie on the reaction path (the IRC) above the branching point [in general this will be the transition state, as in reaction (1), or a saddle point having two imaginary frequencies - a symmetry constrained "transition state" - as in reaction (2)]. Note that, regardless of the coordinate system chosen to map out the reaction path, the initial geometry should be specified in internal coordinates via the Gaussian $82 \mathrm{Z}$ matrix. All 3N-6 variables should be specified for a system with $\mathrm{N}$ atoms, even if some of these are identical or fixed due to symmetry. The algorithm incorporates features which ensure that the symmetry is maintained along the reaction path despite the presence of redundant variables.

3. Set NSIGN to indicate the curvature pattern along the perpendicular (symmetrybreaking) mode during the search: If the curvature changes from positive to negative, NSIGN $=+1$; if negative to positive, NSIGN $=-1$.

4. Evaluate (or read in if already available) the force constant matrix.

5. If the search is in internal coordinates diagonalize the internal-coordinate Hessian; otherwise carry out a normal mode analysis on the cartesian force constant matrix. (At an arbitrary point along the reaction path, project out the gradient before the normal mode analysis.) Mass weight the cartesian coordinates if appropriate. Select and store the IRC and perpendicular modes. On the first step these will generally be the lowest modes that conserve and break symmetry, respectively; on subsequent steps the IRC mode will be the mode which has the greatest overlap with the gradient vector.

6. Check for convergence on the perpendicular mode; if the curvature LAMBDA along this mode is less than HCONV (default $10^{-5}$ ) stop with the current point as the branch point.

7. If LAMBDA is such that the branch point has not been reached, that is, if NSIGN*LAMBDA $>0$, then estimate the distance to the branching point using the quadratic extrapolation step. Store LAMBDA in CP1. If NSIGN*LAMBDA $<0$ then we have overshot the branch point and need to go back. If CP1 is non-zero (i.e., a previous 
Table I. Options available for OPT = BRANCH.

\begin{tabular}{|c|c|c|}
\hline $\begin{array}{l}C \\
C\end{array}$ & Dptions & COMMON/IOP/ \\
\hline $\begin{array}{l}C \\
C \\
C \\
C \\
C\end{array}$ & $\operatorname{IDP}(6)$ & $\begin{array}{l}\text { MAXIMUM NUMBER OF HESSIAN EVALUATIONS ALLOWED } \\
0 \text { MIN( } 15, \text { NVAR }+10 \text { ) } \\
\text { (WHERE NVAR = NUMBER OF VARIABLES) } \\
\text { N N EVALUATIONS }\end{array}$ \\
\hline $\begin{array}{l}\text { C } \\
C \\
C \\
C\end{array}$ & $10 P(7)$ & $\begin{array}{l}\text { CONVERGENCE CRITERION ON ZERO HESSIAN EIGENVALUE } \\
0 \quad 10.0 D-6 \\
N \\
N \\
10.0-\mathrm{N}\end{array}$ \\
\hline $\begin{array}{l}\mathrm{C} \\
\mathrm{C} \\
\mathrm{C} \\
\mathrm{C}\end{array}$ & $\operatorname{IOP}(8)$ & $\begin{array}{ll}\text { MAXIMUM STEPSIZE ALLOWED DURING IRC SEARCH } \\
0 \quad \text { DMAX }=0.15 \\
\mathrm{~N} & \text { DMAX }=0.001 * N\end{array}$ \\
\hline $\begin{array}{l}\mathrm{C} \\
\mathrm{C} \\
\mathrm{C} \\
\mathrm{C}\end{array}$ & IOP(9) & $\begin{array}{lll}\text { MINIMUM STEPSIZE ALLOWED DURING IRC SEARCH } \\
0 & \text { DMIN }=0.005 & \\
\mathrm{~N} & \text { DMIN }=0.001 * \mathrm{~N} & \end{array}$ \\
\hline $\begin{array}{l}C \\
C \\
C \\
C \\
C \\
C \\
C \\
C \\
C\end{array}$ & $\operatorname{IOP}(10)$ & $\begin{array}{ll}\text { INPUT OF INITIAL HESS IAN } \\
\text { ALL VALUES MUST BE IN ATOMIC UNITS } \\
0 \quad \text { CALCULATE HESSIAN ANALYTICALLY } \\
1 & \text { READ IN FULL FRCNST MATRIX } \\
& \text { (LLWER TRIANGLE, FREE FORMAT) } \\
3 & \text { READ FRCNST MATRIX FROM THE CHECKPOINT FILE } \\
5 & \text { READ CARTESIAN FORCE CONSTANTS FROM CHECKPOINT } \\
& \text { FILE }\end{array}$ \\
\hline $\begin{array}{l}C \\
C \\
C \\
C\end{array}$ & $\operatorname{IOP}(11)$ & $\begin{array}{l}\text { MINIMUM NUMBER OF IRC STEPS PER CYCLE } \\
0 \quad 3 \text { STEPS } \\
N \\
N\end{array}$ \\
\hline $\begin{array}{l}\mathrm{C} \\
\mathrm{C} \\
\mathrm{C} \\
\mathrm{C} \\
\mathrm{C} \\
\mathrm{C} \\
\mathrm{C}\end{array}$ & $\operatorname{IOP}(12)$ & $\begin{array}{l}\text { INPUT QF INITIAL IRC SEARCH DIRECTION } \\
0 \text { START SEARCH FROM TS } \\
\text { USE HESSIAN EIGENVECTOR AS SEARCH DIRECTION } \\
\text { (SEe IC arraY fOr EIgEnVeCtor SIgn) } \\
\text { START SEARCH FROM A POINT ON THE IRC } \\
\text { DETERMINE IRC SEARCH DIRECTION FROM GRADIENT }\end{array}$ \\
\hline $\begin{array}{l}\text { C } \\
C \\
C \\
C \\
C\end{array}$ & $\operatorname{IOP}(13)$ & $\begin{array}{ll}\text { SEARCH TYPE } & \\
0 & \text { USE MASS-WE IGHTED CARTES I ANS } \\
1 & \text { USE DRDINARY CARTESIANS } \\
2 & \text { USE INTERNAL CODRDINATES }\end{array}$ \\
\hline $\begin{array}{l}C \\
C \\
C \\
C \\
C\end{array}$ & $\operatorname{IOP}(14)$ & $\begin{array}{ll}\text { PROJECTED GRADIENT FLAG } \\
0 \\
\text { USE PROJECTED GRADIENT TO FIT } \\
\text { PARABOLA DURING LINE SEARCH } \\
1 \text { DO NOT USE. ENERGY SEARCH ONLY }\end{array}$ \\
\hline $\begin{array}{l}\text { C } \\
\mathrm{C} \\
\mathrm{C} \\
\mathrm{C}\end{array}$ & $\operatorname{IOP}(15)$ & $\begin{array}{l}\text { FINITE-DIFFERENCE STEPSIZE } \\
0 \quad 0.005 \\
N \quad 0.0001 * N\end{array}$ \\
\hline
\end{tabular}


Table I. (continued)

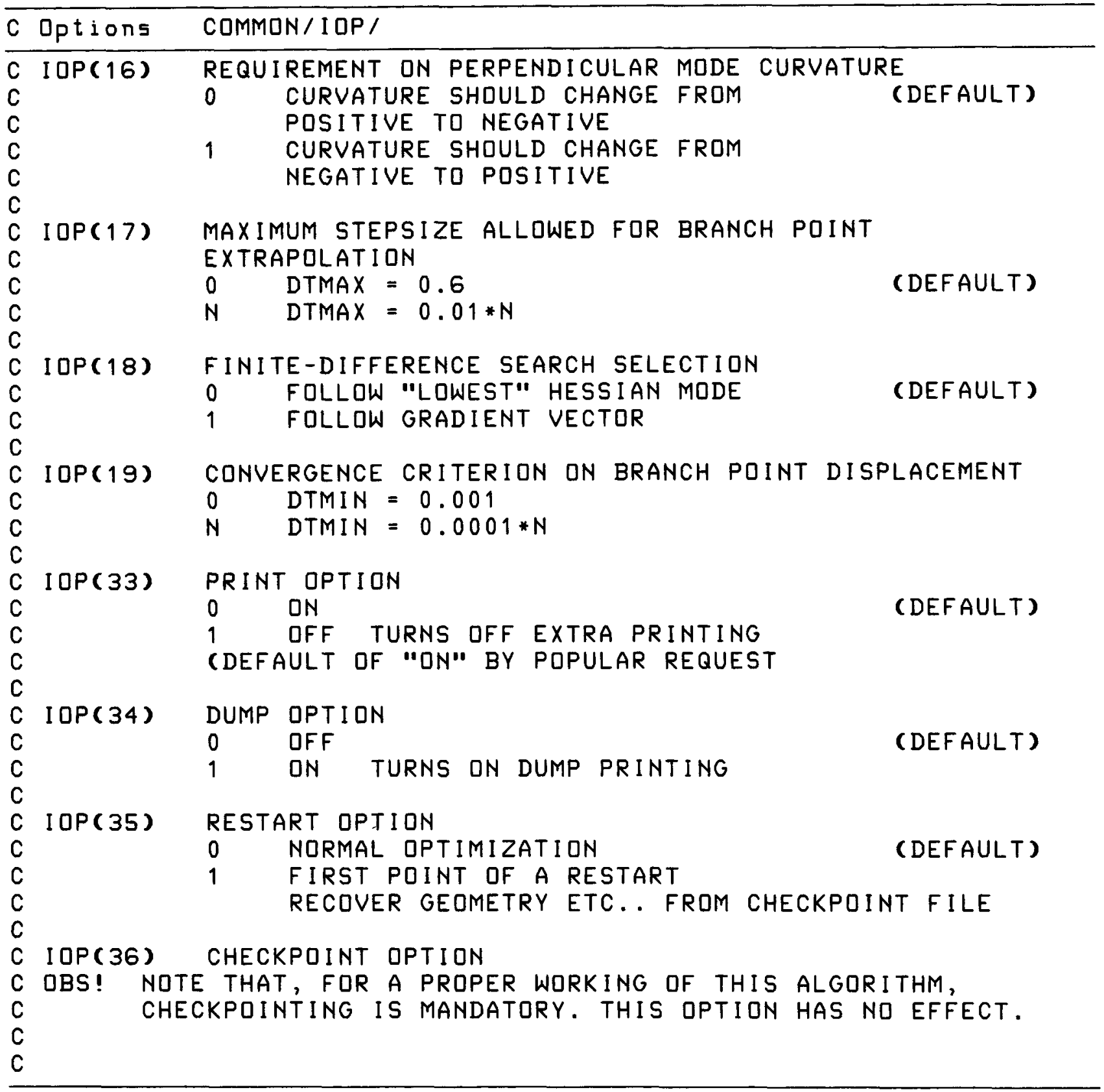

curvature at a point back up the reaction path is known) then linearly interpolate between the two points; if the estimated distance to the branching point is less than the distance to the last point calculated on the path, go back to this point and continue the search; if not, then reject the interpolation in favor of a finitedifference extrapolation. Store LAMBDA in CM1 and save the current point as a marker to restrict the search region.

8. Check for convergence on the branch point displacement; if the estimated distance to the branch point is less than
DTMIN (default 0.001 a.u.) stop with the current point as the branch point.

9. Work out the number of points to be calculated down the reaction path for this cycle; this will depend on the estimated distance to the branch point and the three criteria (1)-(3) given in Section IV.

10. Start the IRC search proper. During the search all points located on the IRC and their corresponding gradients are stored for possible future use in interpolation steps (see 7).

11. When the required distance has been walked down the reaction path, reev- 
aluate the Hessian and, provided the number of Hessian evaluations has not exceeded a predefined limit MAXSTEP, return to step 5.

Within Gaussian 82, the branch point algorithm has been given link number 114; it is invoked by the keyword OPT=BRANCH. A list of the options currently available for the algorithm is given in Table $I$.

\section{AVAILABILITY OF THE CODE}

The FORTRAN code for the above algorithm is available and can be supplied, along with the modifications needed to run in a Gaussian 82 environment, on request. Both VAX and IBM versions exist. All intending users should contact the author (JB) directly. A separate IRC search algorithm for Gaussian 82 users is also available.

One of us (JB) would like to thank Drs. A.J. Stone and J.F. Gaw for useful discussions and S. M. Colwell for bringing ref. 10 to his attention.

\section{References}

1. K. Fukui, J. Phys. Chem., 74, 4161 (1970).

2. K. Ishida, K. Morokuma, and A. Komornicki, J. Chem. Phys., 66, 2153 (1977).

3. K. Muller, Angew. Chem. Int. Ed. (Engl), 19, $1(1980)$.

4. M. V. Basilevsky and A. G. Shamov, Chem. Phys., 60, 347 (1981).

5. D. K. Hoffmann, R.S. Nord, and K. Ruedenberg, Theor. Chim. Acta, 69, 265 (1986).

6. P. Valtazanos and K. Ruedenberg, Theor. Chim. Acta, 69, 281 (1986).

7. E. B. Wilson, Jr., J.C. Delius, and P. C. Cross, Molecular Vibrations, McGraw-Hill, New York 1955.

8. M. Sana, G. Reckinger, and G. Leroy, Theor. Chim. Acta, 58, 145 (1981).

9. A. Tachibana and K. Fukui, Theor. Chim. Acta, 49, 321 (1978).

10. W. H. Miller, N. C. Handy, and J.E. Adams, J. Chem. Phys., 72, 99 (1980).

11. S. M. Colwell, Mol. Phys., 51, 1217 (1984).

12. M.W. Schmidt, M.S. Gordon, and M. Dupuis, J. Am. Chem. Soc., 107, 2585 (1985).

13. J. Baker, J. Comp. Chem., 7, 385 (1986).

14. J. Baker, J. Comp. Chem., 8, 563 (1987).

15. J.S. Binkley, M.J. Frisch, D.J. Defrees, K. Raghavachari, R. A. Whiteside, H. B. Schegel, E.M. Fluder, and J.A. Pople, Carnegie-Mellon University, Pittsburgh, PA 15213, USA. 